\title{
Síndrome Hemolítico-Urêmica Atípica: Manifestações clínicas e desafios no
}

\section{diagnóstico}

\author{
Atypical Hemolytic-Uremic Syndrome: Clinical manifestations and challenges in diagnosis \\ Síndrome Hemolítico-Urémico Atípico: Manifestaciones clínicas y desafíos en el diagnóstico
}

Lennara Pereira Mota ORCID: https://orcid.org/0000-0002-2629-6634 Centro Universitário Maurício de Nassau, Brasil E-mail: lennaramota@hotmail.com

Maria Vitalina Alves de Sousa ORCID: https://orcid.org/0000-0003-4448-2489 Centro Universitário INTA, Brasil E-mail: enf.vitalinaalves@gmail.com

Ana Alinne Gomes Da Penha

ORCID: https://orcid.org/0000-0001-9253-1199

Universidade Regional do Cariri, Brasil

E-mail: anaalinne.nurse@ gmail.com

Alexandra Rodrigues Cardoso

ORCID: https://orcid.org/0000-0002-7277-4668

Centro Universitário Maurício de Nassau, Brasil

E-mail: alexandrarodcar@gmail.com

Francisco De Assis Da Silva Sousa ORCID: https://orcid.org/0000-0002-9665-5284

Hospital Regional do Sertão Central, Brasil E-mail: fassis90assis@gmail.com

Francilene Vieira Da Silva Freitas ORCID: https://orcid.org/0000-0003-3647-1587

Universidade Estadual do Maranhão, Brasil E-mail: francilenev@gmail.com

Josana de Mello Dantas

ORCID: https://orcid.org/0000-0001-6768-656X

Centro Universitário Estácio de Sergipe, Brasil

E-mail: contato@josanamello.com.br

Thaline Ravena Nunes Costa

ORCID: https://orcid.org/0000-0002-8577-4034

Centro Universitário Uninovafapi, Brasil

E-mail: thalineravena1903@gmail.com

Thaynara Alves Rodrigues Paulo da Silva

ORCID: https://orcid.org/0000-0002-8268-9627

Centro Universitário Uninovafapi, Brasil

E-mail: thaynara.alves.biom@gmail.com

Eudes Rufino da Silveira Filho

ORCID: https://orcid.org/0000-0003-0360-1322

Centro Universitário Uninovafapi, Brasil

E-mail: personalgigaeudes@gmail.com

Wilian Reis Rosário

ORCID: https://orcid.org/0000-0003-0187-0502

Universidade Ceuma, Brasil

E-mail: wilianreisr@gmail.com

Regina Célia Da Silva

ORCID: https://orcid.org/0000-0002-6636-5999

Universidade Federal do Piauí, Brasil

E-mail: regina.dasilva@hotmail.com

Lucas Costa de Gois

ORCID: https://orcid.org/0000-0002-2569-5553

Universidade Estadual do Piauí, Brasil

E-mail: goislucas2013@outlook.com

Jaqueline Dantas da Costa

ORCID: https://orcid.org/0000-0003-2570-1949

Centro Universitário Uninovafapi, Brasil

E-mail: jaquedc87@gmail.com 


\author{
Mayara Madeira de Carvalho \\ ORCID: https://orcid.org/0000-0002-0768-3874 \\ Centro de Ensino Unificado de Teresina, Brasil \\ E-mail: mayangel1000@ hotmail.com \\ Stefânia Araújo Pereira \\ ORCID: https://orcid.org/0000-0001-5288-8193 \\ Universidade Estadual do Piauí, Brasil \\ E-mail: stefania.rillys@gmail.com \\ Joice Mara Ferreira Dos Santos \\ ORCID: https://orcid.org/0000-0002-3231-750X \\ Associação de Ensino Superior do Piauí, Brasi \\ E-mail: joicinha2.2kinha@gmail.com
}

\begin{abstract}
Resumo
A síndrome hemolítico-urêmica atípica (SHUa) é caracterizada por uma tríade de trombocitopenia, anemia hemolítica micro angiopática e insuficiência renal aguda. A patogênese da SHU corresponde à micro angiopatia trombótica (MAT). O trabalho tem por objetivo expor através da análise de artigos científicos as principais manifestações clínicas e os desafios no diagnóstico da Síndrome Hemolítico-Urêmica Atípica. Trata-se de uma revisão bibliográfica de caráter qualitativo que se baseia na produção científica a partir de estudos já publicados entre os anos de 2018 a 2021 . Embora a síndrome hemolítico-urêmica atípica seja rara, está relacionada a complicações e sequelas importantes secundárias a alterações da função renal; $42 \%$ das crianças e $27 \%$ dos adultos podem apresentar trombocitopenia grave $(<50.000$ plaquetas $/ \mathrm{mL}$ ). Da mesma forma, a lesão renal aguda relacionada é grave em $59 \%$ das crianças e $81 \%$ dos adultos, e pode ser acompanhada por envolvimento glomerular, creatinina, hematúria, proteinúria, edema e hipertensão. O tratamento que possui mais eficácia contra a SHUa é com a utilização do fármaco Eculizumab. Este fármaco apresenta um alto custo, mas estudos mostram aumento da expectativa e qualidade de vida desses pacientes com o uso desta medicação. É necessário que sejam realizados mais estudos acerca desta doença para o desenvolvimento de novas medidas de diagnóstico e terapias contra a SHUa com menor custo e com fácil acesso para a população devido a gravidade da doença e da sua alta taxa de mortalidade.
\end{abstract}

Palavras-chave: Microangiopatias; Anemia hemolítica; Síndrome urêmica; Diagnóstico.

\begin{abstract}
Atypical hemolytic uremic syndrome (aHUS) is characterized by a triad of thrombocytopenia, microangiopathic hemolytic anemia, and acute renal failure. The pathogenesis of HUS corresponds to thrombotic microangiopathy (THA). The work aims to expose through the analysis of scientific articles the main clinical manifestations and challenges in the diagnosis of Atypical Hemolytic-Uremic Syndrome. This is a literature review of a qualitative nature based on scientific production based on studies published between 2018 and 2021. Although atypical hemolytic uremic syndrome is rare, it is related to complications and important sequelae secondary to alterations of kidney function; $42 \%$ of children and $27 \%$ of adults may have severe thrombocytopenia $(<50,000$ platelets $/ \mathrm{mL})$. Similarly, related acute kidney injury is severe in 59\% of children and $81 \%$ of adults, and may be accompanied by glomerular involvement, creatinine, hematuria, proteinuria, edema, and hypertension. The treatment that is most effective against aHUS is the use of the drug Eculizumab. This drug has a high cost, but studies show an increase in life expectancy and quality of life for these patients with the use of this medication. It is necessary that more studies about this disease are carried out for the development of new diagnostic measures and therapies against aHUS at a lower cost and with easy access for the population due to the seriousness of the disease and its high mortality rate.
\end{abstract}

Key words: Microangiopathies; Hemolytic anemia; Uremic syndrome; Diagnosis.

\title{
Resumen
}

El síndrome urémico hemolítico atípico (SHUa) se caracteriza por una tríada de trombocitopenia, anemia hemolítica microangiopática e insuficiencia renal aguda. La patogenia del SUH corresponde a la microangiopatía trombótica (ATC). El trabajo tiene como objetivo exponer a través del análisis de artículos científicos las principales manifestaciones clínicas y desafíos en el diagnóstico del Síndrome Hemolítico-Urémico Atípico. Se trata de una revisión de la literatura de carácter cualitativo basada en la producción científica basada en estudios publicados entre 2018 y 2021. Aunque el síndrome urémico hemolítico atípico es raro, se relaciona con complicaciones y secuelas importantes secundarias a alteraciones de la función renal; El 42\% de los niños y el 27\% de los adultos pueden tener trombocitopenia grave $(<50000$ plaquetas / ml). De manera similar, la lesión renal aguda relacionada es grave en 59\% de los niños y $81 \%$ de los adultos, y puede estar acompañada de afectación glomerular, creatinina, hematuria, proteinuria, edema e hipertensión. El tratamiento más eficaz contra el SHUa es el uso del fármaco Eculizumab. Este medicamento tiene un costo elevado, pero los estudios muestran un aumento en la esperanza de vida y la calidad de vida de estos pacientes con el uso de este medicamento. Es necesario que se realicen más estudios sobre esta enfermedad para el desarrollo de nuevas medidas diagnósticas y terapias contra el SHUa a menor costo y de fácil acceso para la población debido a la gravedad de la enfermedad y su alta tasa de mortalidad.

Palabras clave: Microangiopatías; Anemia hemolítica; Síndrome urémico; Diagnóstico. 


\section{Introdução}

A síndrome hemolítico-urêmica atípica (SHUa) é caracterizada por uma tríade de trombocitopenia, anemia hemolítica micro angiopática e insuficiência renal aguda. A patogênese da SHU corresponde à micro angiopatia trombótica (MAT). A MAT é um conceito histopatológico, mas é clinicamente caracterizado por anemia, trombocitopenia e falência de órgãos. Na SHU, a doença orgânica é restrita ou dominante no rim, sendo caracterizado pela ativação anormal do complemento, que causa dano endotelial por meio da ativação da hemostasia primária e secundária (Sepúlveda et al., 2018).

A doença geralmente começa de repente. O paciente exibe a tríade SHU característica em que apresenta trombocitopenia, anemia hemolítica não imune e lesão renal aguda. As crianças apresentam palidez, cansaço, recusando-se a comer, vômitos, letargia e, às vezes, edema. Os adultos apresentam fadiga e mal-estar geral (Santos, 2018).

Na fisiopatologia da SHU a protagonista é a MAT sistêmica, que devido a clivagem de C3 e em seguida C5a e da MAC, há consequentemente, esclerose de arteríolas e capilares renais, danos epiteliais, acumulando destruidores do endotélio e aumento dos trombos na luz dos vasos, que tem como consequência uma obstrução desses capilares, que irá provocar lesão renal, causando até mesmo rim fibroso. Essa doença, pode fazer com que o rim perca a sua função de filtragem, que leva o paciente à hemodiálise, e em alguns casos é necessário o transplante. O grande desafio para a resolução da SHU é que mesmo depois em que é realizado o transplante de rim, ocorre uma reativação da doença que irá culminar na destruição do novo rim. Como proteínas mutáveis da SHU são sintetizadas pelo fígado, seria uma opção terapêutica o transplante fígado-rim (Lopes et al., 2020).

Recomenda-se a realização de estudos genéticos para todos os pacientes com suspeita de SHUa ou CAC adicionados. Esses estudos podem confirmar doenças dependentes do complemento, determinar o prognóstico, tratamento, riscos de recorrência e de desenvolver doença renal em estágio terminal, fornece aconselhamento genético familiar e auxilia na tomada de decisão sobre o transplante renal. A biópsia renal confirma a AMT e fornece dados prognósticos, mas isso não é necessário para o diagnóstico, e deve-se considerar que esses pacientes apresentam maior risco de sangramento do que outras doenças renais (Sepúlveda et al., 2018).

Nas últimas décadas, as anormalidades do complemento têm sido associadas a várias doenças do sangue e renais, de modo que os esforços de pesquisa têm aumentado para tentar descrever os mecanismos envolvidos em todas as doenças. Depois de compreender a fisiopatologia, é preciso encontrar ferramentas de diagnóstico e biomarcadores para alvos moleculares para ajudar os pacientes de forma mais eficaz e rápida (Molina et al., 2020).

Algumas pessoas acreditam que o fenômeno MAT atual é o resultado da desregulação da via alternativa do complemento na superfície da célula. Essa alteração pode ser causada por mutações ou polimorfismos que reduzem a atividade de proteínas reguladoras do complemento ou aumentam a função de proteínas ativadoras. Em ambos os casos, a ativação do sistema complemento (induzida por vários gatilhos) não é adequadamente controlada, levando a dano endotelial e trombose (Mena Miranda et al., 2019).

A SHUa é uma patologia considerada rara. A incidência anual é de aproximadamente 2 casos por milhão de adultos e 3,3 casos por milhão de crianças. Esta doença ocorre em qualquer idade, do período neonatal à idade adulta. O início durante a infância ( $\leq 18$ anos) parece ser mais frequente, respondendo por $60 \%$ dos casos, ao contrário da idade adulta, representando $40 \%$. Cerca de $70 \%$ das crianças têm seu primeiro episódio da patologia antes dos dois anos de idade e aproximadamente $25 \%$ em crianças menores de seis meses (Santos, 2018).

O trabalho tem por objetivo expor através da análise de artigos científicos as principais manifestações clínicas e os desafios no diagnóstico da Síndrome Hemolítico-Urêmica Atípica. 


\section{Métodos}

Trata-se de uma revisão bibliográfica de caráter qualitativo que se baseia na produção científica a partir de estudos já publicados. A busca pelos textos foi realizada a partir das seguintes palavras-chaves indexadas no DECS (Descritores em Ciências da Saúde): "Microangiopatias", “Anemia Hemolítica", "Síndrome Urêmica" e "Diagnóstico" na plataforma Scientific Electronic Library Online (SCIELO) e Biblioteca Virtual em Saúde (BVS).

Os critérios de inclusão foram pesquisas científicas publicadas de 2018 a 2021, publicados no idioma português, inglês e espanhol, que atendiam ao problema da pesquisa: Quais as principais manifestações clínicas e dificuldades no diagnóstico da Síndrome Hemolítica-Urêmica atípica? Os critérios de exclusão foram trabalhos científicos com apenas resumos disponíveis, publicações duplicadas, artigos de relato de experiência, reflexivo, editoriais, comentários e cartas ao editor.

A partir do problema de pesquisa foram selecionados artigos publicados em periódicos nacionais e internacionais escolhidos a partir de levantamento realizado por meios dos descritores na biblioteca virtual Scientific Electronic Library Online, Biblioteca Virtual em Saúde (BVS) e dissertações e teses.

Os artigos foram selecionados para leitura e foram incluídos na análise quantitativa artigos que se enquadravam no tema proposto do trabalho.

\section{Resultados e Discussão}

A Figura 1 ilustra os resultados deste trabalho que foram obtidos através da análise quantitativa de publicações sobre as principais manifestações clínicas e dificuldades no diagnóstico da Síndrome Hemolítico-Urêmica atípica nas bases de dados Scientific Electronic Library Online e Biblioteca Virtual em Saúde (BVS). 
Research, Society and Development, v. 10, n. 9, e58210918365, 2021

(CC BY 4.0) | ISSN 2525-3409 | DOI: http://dx.doi.org/10.33448/rsd-v10i9.18365

Figura 1. Fluxograma que apresenta o processo de seleção das publicações sobre Síndrome Hemolítico-Urêmica atípica,

Teresina, Brasil, 2021.

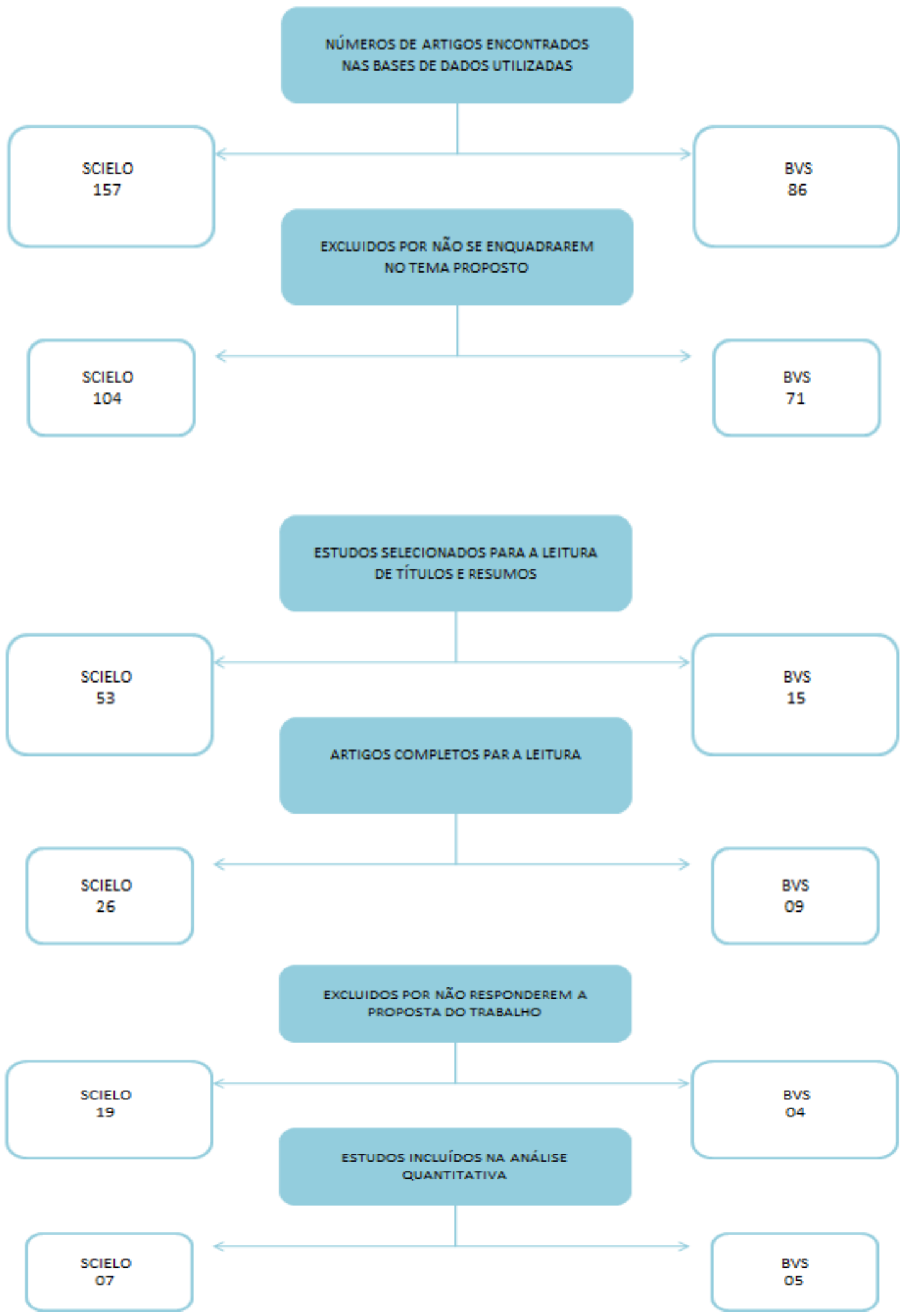

Fonte: Fluxograma elaborado pelos autores. 
Os resultados deste trabalho foram obtidos através da análise quantitativa de publicações sobre Síndrome HemolíticoUrêmica Atípica encontrados nas bases de dados Scientific Electronic Library Online e Biblioteca Virtual em Saúde obtendo 157 artigos na Scielo e 86 artigos na BVS. Foram selecionados para a leitura de títulos e resumos o total de 53 e 15 artigos respectivamente e excluídos 104 artigos da Scielo e 71 da BVS por não se enquadrarem no tema proposto. Foi realizada a leitura completa de 26 artigos da Scielo e 09 artigos da BVS, onde 19 da Scielo e 04 da BVS não responderam a proposta do trabalho. Foram incluídos na análise quantitativa 07 artigos da Scielo e 05 artigos da BVS entre os anos de 2018 a 2021.

De acordo com a pesquisa realizada nas bases de dados, foi possivel listar os principais títulos e objetivos dos estudos publicados na literatura, utilizados neste trabalho conforme o Quadro 1.

Quadro 1. Principais títulos e objetivos dos artigos utilizados na pesquisa, Teresina, Brasil, 2021.

\begin{tabular}{|c|c|c|}
\hline ANO DE PUBLICAÇÃO & TÍTULO & OBJETIVO \\
\hline Sepúlveda et al., 2018. & Síndrome hemolítico urémico atípico. & $\begin{array}{l}\text { Apresentamos uma revisão de SHUa detalhando } \\
\text { sua etiologia, patogênese, apresentação clinica, } \\
\text { diagnóstico e tratamento. }\end{array}$ \\
\hline $\begin{array}{l}\text { Roldán-Tabares; Ruiz-Mejía, } \\
2018 .\end{array}$ & $\begin{array}{l}\text { Síndrome hemolítico urémico atípico: papel del } \\
\text { perfil genético }\end{array}$ & $\begin{array}{l}\text { O objetivo desta revisão é abordar aspectos gerais } \\
\text { da abordagem do paciente com síndrome } \\
\text { hemolítico-urêmica atípica, insistindo nas bases } \\
\text { genéticas desta doença e na utilidade do perfil } \\
\text { genético nesses casos. }\end{array}$ \\
\hline Molina et al., 2020. & $\begin{array}{l}\text { Biomarcadores e alvos moleculares do } \\
\text { complemento no diagnóstico das } \\
\text { microangiopatias trombóticas }\end{array}$ & $\begin{array}{l}\text { Neste trabalho, é apresentada uma revisão do } \\
\text { funcionamento do sistema de complemento em } \\
\text { condições fisiológicas, a fim de explicar } \\
\text { posteriormente quais são as alterações do sistema } \\
\text { compreendidas no desenvolvimento das MAT, e } \\
\text { descrever as ferramentas disponíveis para detectá- } \\
\text { las em laboratório. }\end{array}$ \\
\hline Mena Miranda et al., 2019. & $\begin{array}{l}\text { Avanços no conhecimento da patogêneseda } \\
\text { síndrome hemolítico-urêmica }\end{array}$ & $\begin{array}{l}\text { O objetivo que propomos com a apresentação } \\
\text { deste trabalho é reordenar oconhecimento sobre a } \\
\text { síndrome hemolítico-urêmica em pediatras, que se } \\
\text { deparam com agestão de pacientes com esta } \\
\text { doença em serviços de emergência, hospitalização } \\
\text { eunidades de terapia intensiva. }\end{array}$ \\
\hline Lopes et al., 2020. & $\begin{array}{l}\text { Eculizumab no Transplante Renal: Eficácia na } \\
\text { prevenção da síndrome hemolítica urêmica. }\end{array}$ & $\begin{array}{l}\text { Esse artigo tem como objetivo explicar os } \\
\text { mecanismos de ação do eculizumab, enfatizando } \\
\text { estudos relativos à sua eficácia terapêutica, } \\
\text { principalmente no que diz respeito à Síndrome } \\
\text { Hemolítica Urêmica. }\end{array}$ \\
\hline Santos, 2018. & $\begin{array}{c}\text { Púrpura trombocitopenia trombótica, Síndrome } \\
\text { Hemolítica Urêmica E o Sistema de } \\
\text { Complemento. }\end{array}$ & $\begin{array}{c}\text { O objetivo desta revisão é avaliar o conhecimento } \\
\text { atual a nível fisiopatológico destas três entidades e } \\
\text { enquadrar o sistema de complemento como elo } \\
\text { comum. }\end{array}$ \\
\hline Maximiano et al., 2021. & $\begin{array}{l}\text { Síndrome hemolítica urêmica atípica genética } \\
\text { em crianças: uma experiência de } 20 \text { anos a } \\
\text { partir de um centro terciário. }\end{array}$ & $\begin{array}{l}\text { No presente estudo, objetivamos revisar todos os } \\
\text { casos pediátricos de SHUa admitidos em nosso } \\
\text { centro terciário de nefrologia pediátrica com } \\
\text { identificação de mutação genética causal, nos } \\
\text { últimos } 20 \text { anos, a fim de caracterizar seu perfil } \\
\text { clínico, manejo, resultado em longo prazo, com } \\
\text { foco especial nos episódios de recidiva e } \\
\text { progressão para doença renal crônica (DRC). }\end{array}$ \\
\hline Caetano et al., 2020. & $\begin{array}{l}\text { O caso do eculizumabe: judicialização e } \\
\text { compras pelo Ministério da Saúde. }\end{array}$ & $\begin{array}{l}\text { O estudo examinou as aquisições de eculizumabe, } \\
\text { um anticorpo monoclonal de alto custo utilizado } \\
\text { no tratamento de doenças raras, pelos órgãos }\end{array}$ \\
\hline
\end{tabular}




\begin{tabular}{|c|c|c|}
\hline & & $\begin{array}{l}\text { federais brasileiros, em termos das quantidades } \\
\text { compradas, gastos e preços. }\end{array}$ \\
\hline Bello-Marquez et al., 2020. & $\begin{array}{l}\text { Síndrome nefrótica associada à síndrome } \\
\text { hemolítico-urêmica atípica primária. }\end{array}$ & $\begin{array}{l}\text { Apresentamos aqui um caso de um paciente } \\
\text { pediátrico com síndrome nefrótica associada à } \\
\text { SHU, que foi tratada com eculizumab e mostrou } \\
\text { resposta adequada ao tratamento. }\end{array}$ \\
\hline Tagle et al., 2018. & $\begin{array}{l}\text { Síndrome hemolítico-urêmica atípica tratada } \\
\text { com eculizumabe. Casos Clínicos. }\end{array}$ & $\begin{array}{l}\text { Relatamos duas mulheres de } 17 \text { e } 25 \text { anos com } \\
\text { SHUa catastrófica. Em ambos os casos, fatores de } \\
\text { amplificação do complemento (drogas e } \\
\text { infecções) foram adicionados e agiram sobre uma } \\
\text { vulnerabilidade genética para precipitar a ativação } \\
\text { do complemento e produzir SHUa. }\end{array}$ \\
\hline Chaturvedi et al., 2021. & $\begin{array}{l}\text { Resultados de um protocolo dirigido por um } \\
\text { médico para a descontinuação da terapia de } \\
\text { inibição do complemento na síndrome } \\
\text { hemolítica urêmica atípica. }\end{array}$ & $\begin{array}{c}\text { Aqui, apresentamos um protocolo de } \\
\text { descontinuação e monitoramento de eculizumabe } \\
\text { dirigido por um médico e relatamos uma análise } \\
\text { atualizada dos resultados da descontinuação de } \\
\text { eculizumabe. }\end{array}$ \\
\hline Pugh et al., 2021. & $\begin{array}{l}\text { Intervenções para síndrome hemolítico- } \\
\text { urêmica atípica. }\end{array}$ & $\begin{array}{l}\text { Avaliar os benefícios e malefícios das } \\
\text { intervenções para a SHUa. }\end{array}$ \\
\hline
\end{tabular}

Fonte: SCIELO e BVS.

Desta forma, foi possível listar os principais resultados e conclusões dos estudos publicados na literatura, conforme o Quadro 2.

Quadro 2. Resultados e conclusões dos artigos utilizados, Teresina, Brasil, 2021.

\begin{tabular}{|c|c|c|}
\hline PUBLICAÇÃO & RESULTADOS & CONCLUSÕES \\
\hline Sepúlveda et al., 2018. & $\begin{array}{l}\text { O prognóstico é ruim, } 59 \% \text { das crianças e } 81 \% \\
\text { de adultos requerem diálise no momento de } \\
\text { apresentação e alta taxa de mortalidade, no } \\
\text { período agudo. Foi descrito em } 0,8 \% \text { para } \\
\text { adultos e } 6,7 \% \text { para crianças. Após } 3 \text { a } 5 \text { anos, } \\
50 \% \text { dos bebês e } 70 \% \text { dos adultos estarão } \\
\text { mortos ou dependentes de diálise. }\end{array}$ & $\begin{array}{l}\text { Assim que o diagnóstico de SHUa for } \\
\text { estabelecido ,além de fornecer todas as medidas } \\
\text { de suporte incluindo diálise, se necessário; é } \\
\text { preciso tratar a alteração na via alternativa. Em } \\
\text { casos de MAT secundário, a remoção do agente } \\
\text { causador permite regressão da doença. }\end{array}$ \\
\hline $\begin{array}{l}\text { Roldán-Tabares; Ruiz-Mejía, } \\
2018 .\end{array}$ & $\begin{array}{l}\text { O diagnóstico definitivo da síndrome } \\
\text { hemolítico-urêmica atípica requer confirmação } \\
\text { por teste genético; no entanto, verificou-se que } \\
40 \% \text { dos pacientes não apresentam qualquer } \\
\text { alteração genética conhecida. Além disso, no } \\
\text { momento, esses testes genéticos não fazem parte } \\
\text { do diagnóstico inicial.. }\end{array}$ & $\begin{array}{l}\text { A síndrome hemolítica urêmica atípica é uma } \\
\text { condição rara, mas sem diagnóstico precoce e } \\
\text { tratamento imediato e está associada a } \\
\text { complicações significativas. É importante } \\
\text { identificar quais pacientes com microangiopatia } \\
\text { trombótica estão em risco para essa condição e } \\
\text { são elegíveis para um perfil genético, pois esta é } \\
\text { a única ferramenta diagnóstica que permite } \\
\text { diferenciar totalmente a síndrome hemolítica } \\
\text { urêmica atípica de outras causas de } \\
\text { microangiopatia trombótica. }\end{array}$ \\
\hline Molina et al., 2020. & $\begin{array}{c}\text { O desenvolvimento da tecnologia de } \\
\text { sequenciamento alto desempenho ou } \\
\text { "sequenciamento de próxima geração" } \\
\text { (NGS)permitiu que avanços extremamente } \\
\text { rápidos fossem feitos, auxiliando na busca por } \\
\text { variantes genéticas associadas a doenças raras e } \\
\text { multifatoriais. Patologias como MAT estão entre } \\
\text { as entidades que foram beneficiadas desta } \\
\text { revolução genômica, dado que a presença de } \\
\text { anormalidades em vários genes já foram } \\
\text { descritos em associação com formas hereditárias }\end{array}$ & $\begin{array}{c}\text { O sistema de complemento é um dos sistemas de } \\
\text { defesa que desempenha um papel fundamental } \\
\text { na imunidade. Uma maior compreensão sobre o } \\
\text { sistema complemento com a descrição de } \\
\text { proteínas envolvidas e suas diferentes funções, } \\
\text { permitem descrever parte dos mecanismos } \\
\text { patológicos. }\end{array}$ \\
\hline
\end{tabular}




\begin{tabular}{|c|c|c|}
\hline & da doença. & \\
\hline Mena Miranda et al., 2019. & $\begin{array}{c}\text { Os dados sobre a apresentação clínica de SHUa } \\
\text { são limitados devido ao número depacientes. A } \\
\text { doença atinge crianças e adultos, com casos } \\
\text { relatados desde o períodoneonatal. A } \\
\text { distribuição masculina: feminina é igualitária na } \\
\text { infância, mas tende apredominam nas mulheres } \\
\text { na vida adulta. }\end{array}$ & $\begin{array}{l}\text { Síndrome hemolítico-urêmica (típica ou atípica) } \\
\text { é uma das principais causas de falha lesão renal } \\
\text { aguda em crianças. É caracterizada por uma } \\
\text { tríade consistente de anemia hemolítica. }\end{array}$ \\
\hline Lopes et al., 2020. & $\begin{array}{c}\text { A deposição capilar de anticorpos do } \\
\text { complemento é onde atua o eculizumab. A } \\
\text { deposição de componentes do complemento } \\
\text { C4d-a é o marcador de rejeição de transplante. } \\
\text { Dessa forma o eculizumab vai bloquear a } \\
\text { ativação da via clássica do complemento como } \\
\text { uma tentativa de inibir a deposição e a } \\
\text { destruição do endotélio capilar renal. O } \\
\text { eculizumab, com seu mecanismo de ação } \\
\text { inibitório, diminui a hemólise vascular mediada } \\
\text { pela deposição de C5a, e reduzindo os efeitos } \\
\text { inflamatórios e citolíticos. }\end{array}$ & $\begin{array}{l}\text { Na revisão sistemática de literatura, foram } \\
\text { observadas relevantes melhorias do prognóstico } \\
\text { de pacientes transplantados fazendo prevenção e } \\
\text { tratamento com eculizumab, enfatizando sua } \\
\text { importância para os portadores da SHU. }\end{array}$ \\
\hline Santos, 2018. & $\begin{array}{l}\text { As mutações no CFH são as mais frequentes na } \\
\text { SHUa, com cerca de } 20 \text { a } 30 \% \text { de casos de } \\
\text { SHUa. Mais de } 50 \% \text { das mutações localizam-se } \\
\text { no SCR 19-20. Estas mutações reduzem a } \\
\text { capacidade do CFH para se ligar ao C3b nas } \\
\text { superfícies celulares, inibindo a degradação de } \\
\text { C3b e C3 convertase. Cerca de } 100 \text { mutações } \\
\text { foram descritas no CFH, e aproximadamente } \\
75 \% \text { são heterozigóticas. Algumas mutações, } \\
\text { denominadas de tipo 1, estão associadas com } \\
\text { défice quantitativo no CFH, com baixos níveis } \\
\text { plasmáticos, no entanto, a maioria das mutações } \\
\text { no SCR19-20, relacionam-se com níveis } \\
\text { normais de CFH e deficiência funcional, } \\
\text { denominada mutação de tipo } 2 \text {. }\end{array}$ & $\begin{array}{l}\text { A PTT, STEC-SHU e SHUa são } \\
\text { patologicamente indistinguíveis, no entanto, } \\
\text { apresentam diferentes mecanismos } \\
\text { fisiopatológicos, respetivamente, deficiência de } \\
\text { ADAMTS13, infeção por microorganismos } \\
\text { produtores de shigatoxina e desregulação da via } \\
\text { alternativa do complemento. Contudo, através de } \\
\text { estudos realizados, foi evidenciada ativação do } \\
\text { complemento nestas três entidades, } \\
\text { demonstrando um via comum na sua patogénese. } \\
\text { O Eculizumab é um anticorpo monoclonal que } \\
\text { tem como alvo C5, aprovado para a SHUa. }\end{array}$ \\
\hline Maximiano et al., 2021. & $\begin{array}{l}\text { Quatro pacientes (80\%) foram associados a } \\
\text { anomalias do FHC: um paciente apresentou } \\
\text { mutações heterozigotas no FHC, um paciente } \\
\text { apresentou autoanticorpos contra o FHC } \\
\text { associados à deleção da proteína } 3 \text { e } 1 \\
\text { relacionada ao FHCR (FHCR3/1), e dois } \\
\text { pacientes carregavam uma nova forma de } \\
\text { mutação que não havia sido descrita até o } \\
\text { momento, uma delas incluindo uma única forma } \\
\text { de mutação do FHC e outra com mutação do } \\
\text { FHC associada à mutação do gene DGKe, } \\
\text { ambas homozigotas; um paciente carregava uma } \\
\text { mutação na C3. }\end{array}$ & $\begin{array}{c}\text { Identificar, descrever e compreender as } \\
\text { anomalias genéticas associadas a esta doença } \\
\text { certamente permitirá melhorias importantes em } \\
\text { termos de manejoda doença e do desfecho dos } \\
\text { pacientes. }\end{array}$ \\
\hline Caetano et al., 2020. & $\begin{array}{l}\text { Todas as aquisições por órgãos federais foram } \\
\text { realizadas pelo Ministério da Saúde. As compras } \\
\text { se iniciaram em } 2009 \text {, sendo efetuadas por } \\
\text { dispensa de licitação e para atendimento de } \\
\text { demanda judicial. Houve tendência crescente no } \\
\text { número de compras e quantidades adquiridas ao } \\
\text { longo do tempo. Foram realizadas } 283 \text { compras, } \\
\text { totalizando } 116.792 \text { unidades adquiridas, } 28,2 \% \\
\text { compradas em } 2018 \text {. }\end{array}$ & $\begin{array}{l}\text { O eculizumabe representou gastos extremamente } \\
\text { significativos para o Ministério da Saúde no } \\
\text { período. Todas as compras foram feitas para } \\
\text { atendimento de demandas judiciais, fora do } \\
\text { ambiente competitivo. Seu registro promoveu } \\
\text { queda importante nos preços praticados. O } \\
\text { estudo aponta a relevância do registro sanitário e } \\
\text { da necessidade de monitoramento e auditoria } \\
\text { permanentes das compras de medicamentos para } \\
\text { atendimento de demandas judiciais. }\end{array}$ \\
\hline Bello-Marquez et al., 2020. & $\begin{array}{l}\text { As mutações mais frequentes na população } \\
\text { pediátrica com SHUa são as dos genes CFH, } \\
\text { CFI e MCP. Estes são conhecidos em pacientes } \\
\text { com mutações no CFH que apresentam }\end{array}$ & $\begin{array}{c}\text { Em conclusão, relatamos um paciente pediátrico } \\
\text { que apresentou SHUa e síndrome nefrótica } \\
\text { simultaneamente com comprometimento } \\
\text { hematológico, renal, neurológico e }\end{array}$ \\
\hline
\end{tabular}




\begin{tabular}{|c|c|c|}
\hline & $\begin{array}{l}\text { manifestações mais graves, pior prognóstico e } \\
\text { maior risco de morte ou DRT; } 30 \% \text { destes } \\
\text { ocorrem durante o primeiro episódio, mas esse } \\
\text { risco pode aumentar até } 60 \% \text { durante o primeiro } \\
\text { ano de evolução. }\end{array}$ & $\begin{array}{c}\text { cardiovascular; ele foi tratado com eculizumab } \\
\text { com remissão completa do comprometimento } \\
\text { sistêmico sem a necessidade de uso de } \\
\text { corticosteróides ou outras drogas } \\
\text { imunossupressoras. }\end{array}$ \\
\hline Tagle et al., 2018. & $\begin{array}{l}\text { O prognóstico para SHUa é terrível. A } \\
\text { mortalidade varia entre } 0,8-15 \% \text { no evento } \\
\text { agudo } 7,8 \text { e após } 3 \text { a } 5 \text { anos, 50\% dos bebês e } \\
70 \% \text { dos adultos morrerão ou necessitarão de } \\
\text { TRS } 8,10 \text {. Mutações em CFH, CFHR (genes } \\
\text { relacionados ao CFH), C'3 e CFB (fator de } \\
\text { complemento B) têm pior prognóstico. Ao } \\
\text { contrário, as mutações MCP (proteína cofator de } \\
\text { membrana) têm múltiplas recorrências, com } \\
\text { bom prognóstico e reprodução mínima no } \\
\text { transplante renal. }\end{array}$ & $\begin{array}{l}\text { Dois casos de SHUa são apresentados para } \\
\text { informar a comunidade médica sobre esta } \\
\text { doença rara: sua apresentação clínica, } \\
\text { prognóstico nefasto, necessidade de diagnóstico } \\
\text { oportuno e a possibilidade de mudar sua história } \\
\text { natural graças ao tratamento com Eculizumab. }\end{array}$ \\
\hline Chaturvedi et al., 2021. & $\begin{array}{l}\text { Além da recidiva, os resultados renais de longo } \\
\text { prazo são um ponto final crítico em estudos que } \\
\text { avaliam estratégias restritivas de eculizumabe } \\
\text { (ou ravulizumabe) em TMA mediada por } \\
\text { complemento. Pelo menos } 1 \text { série relatou uma } \\
\text { tendência de diminuição da função renal ao } \\
\text { longo do tempo após a interrupção. }\end{array}$ & $\begin{array}{l}\text { Em resumo, mostramos que para a maioria dos } \\
\text { pacientes com SHUa, a descontinuação da } \\
\text { terapia com inibidor de C5 (eculizumabe) é } \\
\text { segura e aceitável sem perda da função renal } \\
\text { quando feita sob monitoramento próximo para } \\
\text { permitir a detecção precoce e o tratamento de } \\
\text { recidivas. }\end{array}$ \\
\hline Pugh et al., 2021. & $\begin{array}{l}\text { Melhorias substanciais foram observadas na taxa } \\
\text { de filtração glomerular estimada e na qualidade } \\
\text { de vida relacionada à saúde em estudos com } \\
\text { eculizumabe e ravulizumabe. Eventos adversos } \\
\text { graves ocorreram em } 42 \% \text { dos pacientes, e } \\
\text { infecção meningocócica ocorreu em dois } \\
\text { pacientes, ambos tratados com eculizumabe. }\end{array}$ & $\begin{array}{l}\text { Quando comparada com dados históricos, a } \\
\text { inibição terminal do complemento parece } \\
\text { oferecer resultados favoráveis em pacientes com } \\
\text { SHUa, com base em evidências de qualidade } \\
\text { muito baixa extraídas de cinco estudos de braço } \\
\text { único. }\end{array}$ \\
\hline
\end{tabular}

Fonte: SCIELO e BVS.

Baseando-se nos resultados e conclusões dos artigos utilizados, pode-se observar que a SHUa é uma patologia da via alternativa do complemento, considerada primária quando o defeito é hereditário. A causa secundária de SHUa pode ativar o complemento sozinho ou pode atuar como uma condição de amplificação do complemento (CAC) na suscetibilidade genética. Em outras palavras, para que a MAT se desenvolva, é necessário cruzar um "limiar" que desencadeia a ativação da hemostasia intravascular. Se essa ativação se manifestar principalmente com lesão renal, é SHUa. Se a causa for mediada apenas por defeitos genéticos no complemento, o diagnóstico é SHUa primário. Se a causa exclusiva for outra condição (como CAC), será um SHUa secundário. No entanto, o "limiar" da MAT pode ser alcançado pela soma dos defeitos genéticos e do CAC, que é a forma mais comum na prática clínica (SHUa primário mais CAC) (Sepúlveda et al., 2018).

O sistema complemento é uma importante ferramenta para a resposta imune inata e uma ponte entre a imunidade inata e a adquirida, sendo composto por várias proteínas sintetizadas principalmente no fígado. Eles são encontrados como precursores inativos (pró-enzimas) no plasma e na superfície das células (Molina et al., 2020).

O corpo humano tem uma variedade de mecanismos de defesa para resistir a microrganismos, o sistema complemento é um fator-chave da imunidade inata, ajuda na rápida identificação e eliminação de patógenos. O sistema complemento é composto por mais de 30 tipos de proteínas, muitas das quais são sintetizadas no fígado e distribuídas no plasma e superfície celular. As principais funções do sistema incluem iniciar e mediar à resposta inflamatória e participar da remoção de produtos de degradação endógenos, marcar microrganismos patogênicos marcantes para a opsonização e eliminar microrganismos com o complexo de ataque à membrana (MAC). O sistema complemento é ativado por três vias: a via clássica, a lectina e a via alternativa. Essas três são capazes de convergir na clivagem do fator C3 do complemento (Santos, 2018).

A síndrome hemolítico urêmica atípica é quase sempre causada por um ou mais defeitos na regulação da via 
alternativa do complemento, que é causada por anormalidades genéticas em $60 \%$ dos pacientes ou adquirida nos fatores reguladores ou ativadores desta via; mas em alguns pacientes as mutações podem não estar diretamente relacionadas ao sistema complemento, entre as quais destacam-se as enzimas diacilglicerol quinase, plasminogênio e fator de coagulação XII (Roldán-Tabares; Ruiz-Mejía, 2018).

O diagnóstico de SHUa primária no contexto da MAT é um dos diagnósticos de exclusão. O CAC deve ser estudado e outras causas de MAT que são manifestadas por SHU, mas não mediadas por alterações do complemento, devem ser excluídas: drogas, tumores, infecções, PTT, SHU-ECTS e deficiência de cobalamina C. A gravidez está associada a púrpura trombocitopênica trombótica (PTT) e puerpério a SHUa (Sepúlveda et al., 2018).

Em SHU atípico (SHUa), a causa da MAT é a ativação anormal da via alternativa do complemento. Esta ativação pode ser secundária a patologias que exacerbam a atividade do complemento (infecção, pancreatite, gravidez / puerpério, hipertensão maligna, doenças autoimunes, drogas, isquemia, transplante de órgãos, tumores) e / ou alterações genéticas em certos componentes da via alternativa do complemento. Para o desenvolvimento de SHUa, os fatores de amplificação do complemento são combinados em vulnerabilidade genética, portanto, quando o "limiar" for excedido, o MAT será acionado (Tagle et al., 2018).

SHU é uma doença rara e grave com a tríade clássica de anemia hemolítica, trombocitopenia e lesão renal (Pugh et al., 2021). Historicamente, o termo inclui duas doenças: SHU típica associada a agentes infecciosos e SHU atípica associada a vias alternativas não controladas do complemento. No entanto, atualmente é recomendado o uso de uma classificação SHU mais ampla com base no evento desencadeante que levou à apresentação clínica. Portanto, É importante a triagem de cobalamina C, infecções pneumocócicas ou virais (HIV, hepatite A e C, citomegalovírus, influenza H1N1, Epstein-Barr), câncer, drogas ou transplante de órgãos sólidos (como rim) ou células hematopoiéticas (Molina et al., 2020).

A SHU atípica deve ser suspeitada em pacientes menores de 6 meses, com mais de um episódio, história familiar de SHU, níveis baixos de C3 e recorrência após transplante renal. Ao contrário do SHU-ECTS, que geralmente é um evento único, a SHUa torna-se uma entidade crônica devido à origem genética da doença e tem um prognóstico ruim. Após o primeiro ataque de SHUa, a taxa de mortalidade é de 10-15\% e até 50\% dos pacientes não recuperam a função renal (Mena Miranda et al., 2019).

A SHU é uma doença genética sistêmica, de caráter crônico e progressivo, causada por mutações das proteínas do sistema complemento que levam à perda dos inibidores naturais desse sistema, culminando na sua ativação crônica e na produção do componente C5a e do complexo de ataque à membrana (MAC). A doença pode ser típica, que tem um melhor prognóstico, e está associada à infecção por E. Coli produtores da Toxina Shiga-like, já a atípica, tem um pior prognóstico, e possui diversos fatores que podem estar associados, como a hiperativação do complemento, doenças imunossupressoras, infecções por Pneumococcus (Lopes et al., 2020).

Vários estudos determinaram que 40-60\% dos pacientes com SHU carregam mutações nos genes do complemento (gene do fator $\mathrm{H}$ do complemento [CFH], proteína do cofator de membrana [MCP], gene do fator I do complemento [CFI], trombomodulina [THBD], gene do fator B do complemento (CFB) e gene C3, que estão relacionados à desregulação da via alternativa. O FH atua no plasma para controlar a homeostase do complemento e a superfície celular para prevenir danos aos componentes. A mutação FH está associada ao agrupamento SHUa na região C-terminal e reduz a proteção da superfície celular de danos acidentais causados pela ativação do complemento, mas não afeta a regulação do complemento no plasma (Mena Miranda et al., 2019).

Embora a síndrome hemolítico-urêmica atípica seja rara, está relacionada a complicações e sequelas importantes secundárias a alterações da função renal; $42 \%$ das crianças e $27 \%$ dos adultos podem apresentar trombocitopenia grave (<50.000 plaquetas / mL). Da mesma forma, a lesão renal aguda relacionada é grave em 59\% das crianças e $81 \%$ dos adultos, e 
pode ser acompanhada por envolvimento glomerular, creatinina, hematúria, proteinúria, edema e hipertensão. Além disso, de 20 a 48\% dos pacientes podem ter manifestações extra-renais, como alterações no estado mental, convulsões, pancreatite, diarreia e hepatite (Roldán-Tabares; Ruiz-Mejía, 2018).

Nos últimos anos, foi descoberto que a SHUa é relacionada com anormalidades genéticas ou autoimunes que levam à desregulamentação da via alternativa do complemento que está na superfície do endotélio vascular. Em quase $60 \%$ dos pacientes com SHUa, são relatadas mutações em genes codificadores de proteínas que regulam o complemento. Foram associadas à SHUa mutações em 6 genes, fator H do complemento (FHC), fator B do complemento, fator I do complemento, proteína cofator de membrana (PCM), C3 e trombomodulina (Maximiano et al., 2021).

Infelizmente, não existe atualmente um teste definitivo para determinar um diagnóstico preciso da síndrome hemolítico-urêmica atípica, razão pela qual é considerada um diagnóstico de exclusão, porque diante da suspeita clínica de microangiopatias trombóticas, outras causas mais comuns, como a síndrome hemolítica e a púrpura trombocitopênica trombótica urêmica típica devem ser primeiro descartadas. A fim de determinar potenciais mutações genéticas, testes genéticos caros são necessários; no entanto, essa característica genética não só tem significado diagnóstico, mas também tem grande valor prognóstico. Prescrições de tratamento adequadas podem reduzir significativamente as taxas de morbidade e mortalidade (Roldán-Tabares; Ruiz-Mejía, 2018).

Até 2011 a Plasmaférese era o tratamento de escolha para a SHUa, em que era utilizada a dose de 1,5 volume de plasma por sessão com plasma fresco congelado. O objetivo deste tratamento era de remover fatores de complemento mutados e a presença de autoanticorpos, fornecendo fatores do sistema complemento normais e promover remissão hematológica. Esse tratamento não apresenta melhora significativa da função renal dos pacientes, e os portadores de SHUa apresentam alta morbimortalidade no seguimento de um ano (Bello-Marquez et al., 2020).

O eculizumab é um fármaco (anticorpo monoclonal humanizado) que foi desenvolvido e possui a capacidade de inibir a via terminal do complemento, sendo uma alternativa mais viável para o paciente no ponto de vista de que não é uma terapia invasiva e não possui risco de infecção e dificuldade, pois não é necessário encontrar doador e dois órgãos compatíveis (Lopes et al., 2020).

O Eculizumab é um medicamento de alto custo usado para aliviar complicações de adultos e crianças com hemoglobinúria paroxística noturna (HPN) e síndrome hemolítica urêmica atípica (SHUa). A SHUa é uma entidade clínica que se manifesta como trombocitopenia, anemia hemolítica micrangiopática e lesão renal progressiva causada por mutações em genes que controlam o sistema complemento. É considerada uma doença rara, pois afeta cerca de 65 pessoas a cada 100 mil (Caetano et al., 2020).

$\mathrm{Na}$ era pré-eculizumabe, $56 \%$ dos pacientes com SHUa progrediram para doença renal em estágio terminal no primeiro ano. O eculizumabe é um anticorpo monoclonal anti-C5 muito eficaz no tratamento da SHUa e leva à melhora rápida e contínua dos parâmetros hematológicos e da função renal (Chaturvedi et al., 2021).

\section{Conclusão}

A Síndrome Hemolítico-Urêmico Atípica é uma doença considerada rara, mas que apresenta um mau prognóstico. Ocorre devido à ativação anormal do sistema complemento, e consequentemente apresenta uma tríade de anemia hemolítica, trombocitopenia e insuficiência renal. É uma patologia que não possui um diagnóstico específico, sendo realizado geralmente a partir de exclusão de outras doenças. Existem testes genéticos mais eficazes no diagnostico, porém esses testes possuem alto custo e não são utilizados com frequência. O tratamento que possui mais eficácia contra a SHUa é com a utilização do fármaco Eculizumab. 
Este fármaco apresenta um alto custo, mas estudos mostram aumento da expectativa e qualidade de vida desses pacientes com o uso desta medicação. É necessário que sejam realizados mais estudos acerca desta doença para o desenvolvimento de novas medidas de diagnóstico e terapias contra a SHUa com menor custo e com fácil acesso para a população devido a gravidade da doença e da sua alta taxa de mortalidade.

\section{Referências}

Bello-Marquez, D. C., Nieto-Rios, J. F., Serna-Higuita, L. M., \& Gonzalez-Vergara, A. J. (2020). Síndrome nefrótica associada à síndrome hemolítico-urêmica atípica primária. Brazilian Journal of Nephrology, (AHEAD).

Caetano, R., Rodrigues, P. H. A., Corrêa, M. C. V., Villardi, P., \& Osorio-de-Castro, C. G. S. (2020). O caso do eculizumabe: judicialização e compras pelo Ministério da Saúde. Revista de Saúde Pública, 54, 22.

Chaturvedi, S., Dhaliwal, N., Hussain, S., Dane, K., Upreti, H., Braunstein, E. M., \& Brodsky, R. A. (2021). Resultados de um protocolo dirigido por um médico para a descontinuação da terapia de inibição do complemento na síndrome hemolítica urêmica atípica. Blood Advances , 5 (5), $1504-1512$.

Lopes, A. J. B., Magalhães, B. A., Bezerra, F. C. M., Megali, H. A., de Souza Queiroz, H., de Oliveira, M. T., \& Silva, T. P. (2020). Eculizumab no Transplante Renal: Eficácia na prevenção da síndrome hemolítica urêmica. Brazilian Journal of Health Review, 3(4), 9890-9896.

Maximiano, C., Silva, A., Duro, I., Branco, T., Correia-Costa, L., Teixeira, A., \& Afonso, A. C. (2021). Síndrome hemolítica urêmica atípica genética em crianças: uma experiência de 20 anos a partir de um centro terciário. Brazilian Journal of Nephrology.

Mena Miranda, V. R., \& Puig Souza, L. (2019). Avances en el conocimiento de la patogénesis del síndrome hemolítico urémico. Revista Cubana de Pediatría, $91(3)$

Molina, N. M., Rotondo, S., Dos Santos, C., \& Sánchez-Luceros, A. (2020). Biomarcadores y blancos moleculares del complemento en el diagnóstico de las microangiopatías trombóticas. Acta Bioquímica Clínica Latinoamericana, 54(4), 437-453.

Pugh, D., D O'Sullivan, E., Duthie, F. A., Masson, P., \& Kavanagh, D. (2021). Interventions for atypical haemolytic uraemic syndrome. Cochrane Database of Systematic Reviews, (3)

Roldán-Tabares, M. D., \& Ruiz-Mejía, C. (2018). Síndrome hemolítico urémico atípico: papel del perfil genético. Medicina interna de México, 34(3), 394402 .

Santos, M. M. D. A. (2018). Púrpura trombocitopénica trombótica: síndrome hemolítica urémica e o sistema de complemento (Doctoral dissertation).

Sepúlveda, R. A., Tagle, R., \& Jara, A. (2018). Síndrome hemolítico urémico atípico. Revista médica de Chile, 146(6), 770-779.

Tagle, R., Rivera, G., Walbaum, B., \& Sepúlveda, R. A. (2018). Síndrome hemolítico urémico atípico en tratamiento con eculizumab. Casos clínicos. Revista médica de Chile, 146(2), 254-259. 